\title{
Infección diseminada por Strongyloides stercoralis en dos receptores de trasplante renal de un único donante
}

\section{Disseminated Strongyloides stercoralis infection in two persons receiving transplanted kidneys from a single donor: reported cases}

Correspondencia Enrique Paz Rojas

epaz@clinicajavierprado.com.pe

Recibido: 23/04/2017

Arbitrado por pares

Aprobado: 02/08/2017

Citar como: Paz Rojas E, Cerrón Cabezas C, Cruz Touzet J, Delgado Gonzales V, Gonzales-Hamada L, Maguiña Vargas $C$, et al. Infección diseminada por Strongyloides stercoralis en dos receptores de trasplante renal de un único donante. Acta Med Peru. 2017;34(3):225-30
Enrique Paz Rojas ${ }^{1}$, Carmen Cerrón Cabezas ${ }^{1}$, José Cruz Touzet ${ }^{1}$, Víctor Delgado Gonzales², Liliana Gonzales-Hamada ${ }^{3}$, Ciro Maguiña Vargas ${ }^{4}$, Wilfredo Flores Paredes ${ }^{5}$

1 Servicio de Cuidados Intensivos, Departamento de Cuidados Críticos, Hospital Nacional Guillermo Almenara Yrigoyen EsSalud. Lima, Perú.

2 Servicio de Patología Quirúrgica, Hospital Nacional Guillermo Almenara Yrigoyen, EsSalud. Lima, Perú.

3 Departamento de trasplantes, Hospital Nacional Guillermo Almenara Yrigoyen, EsSalud. Lima, Perú.

4 Departamento de Enfermedades Infecciosas, Tropicales y Dermatológicas, Hospital Nacional Cayetano Heredia. Lima, Perú.

5 Servicio de Microbiología. Hospital Nacional Guillermo Almenara Yrigoyen. Lima, Perú.

\section{RESUMEN}

La infección por Strongyloides stercoralis a partir de donantes de órganos es infrecuente, pero cuando se presenta genera diversas complicaciones. Se reportan dos casos de estrongiloidiasis diseminada derivado de un único donante cadavérico en dos receptores de trasplante renal. Ambos pacientes se presentaron con síntomas gastrointestinales y síntomas respiratorios más lesiones dermatológicas en región abdominal. El diagnóstico se basó en el hallazgo de larvas en lavado broncopulmonar, lesiones dermatológicas, y aspirado duodenal. A pesar del manejo con albendazol e ivermectina por vía oral el curso clínico fue fatal en uno de ellos. El segundo paciente recibió precozmente ivermectina por vía subcutánea y sobrevivió con falla intestinal persistente.

Palabras clave: Strongyloides stercoralis; Trasplante de riñón; Donante de órgano (fuente: DeCS BIREME).

\section{ABSTRACT}

AStrongyloides stercoralis infection acquired from the donor in a transplant receptor is not a common occurrence, but its complications have been well known. We report two cases of disseminated strongyloidiasis derived from a single cadaveric donor at two renal transplant recipients. Both patients presented gastrointestinal and respiratory symptoms plus dermatological lesions in the abdominal region. The diagnosis was based on the finding of larvae in bronchopulmonary lavage, dermatological lesions, and duodenal aspiration. Despite the oral administration of albendazole and ivermectin, the clinical course was fatal in one of them. The second patient received precociously ivermectin by subcutaneous administration and survived with persistent bowel failure.

Keywords: Strongyloides stercoralis; Kidney transplantation; Tissue donors (source: MeSH NLM). 


\section{INTRODUCCIÓN}

La estrongiloidiasis es una enfermedad parasitaria causada por el nematodo Strongyloides stercoralis que afecta entre 30 a 100 millones de personas en el mundo. Las complicaciones, están asociadas con el alcoholismo, los trasplantes de órganos, y el virus HTLV-1, que sumados a un diagnóstico tardío pueden conducir a la muerte del paciente ${ }^{[1]}$. Este nematodo intestinal es endémico y se encuentra ampliamente diseminado en áreas tropicales y subtropicales ${ }^{[2,3]}$. La hembra adulta es muy pequeña y casi transparente, mide aproximadamente 2,2-2,5 mm de largo con un diámetro de $50 \mu \mathrm{m}$, habitando en espacios formados entre los enterocitos del intestino delgado. Por su parte, el macho se caracteriza por no ser un parásito intratisular ${ }^{[3]}$.

Una infección diseminada casi siempre es el resultado de una hiperinfección, donde se pueden encontrar larvas en cualquier tejido o secreción, particularmente en esputo y en la piel. La sintomatología cursa con anemia, dolor abdominal,

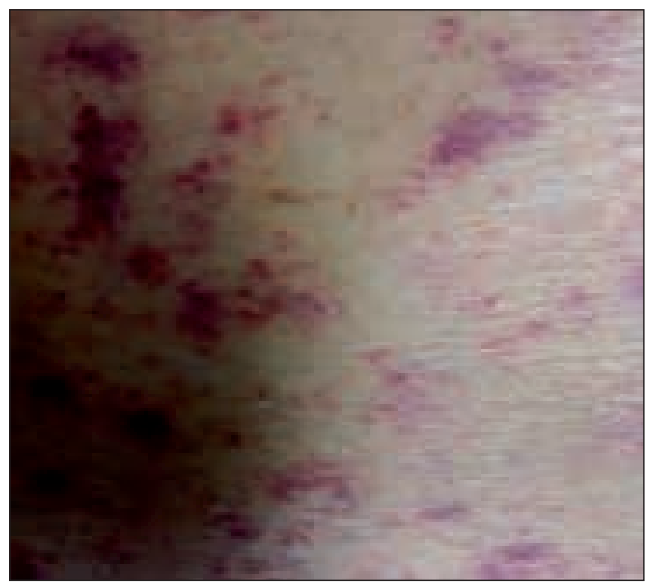

Figura 1. Caso 1: se observan lesiones purpúricas en abdomen.

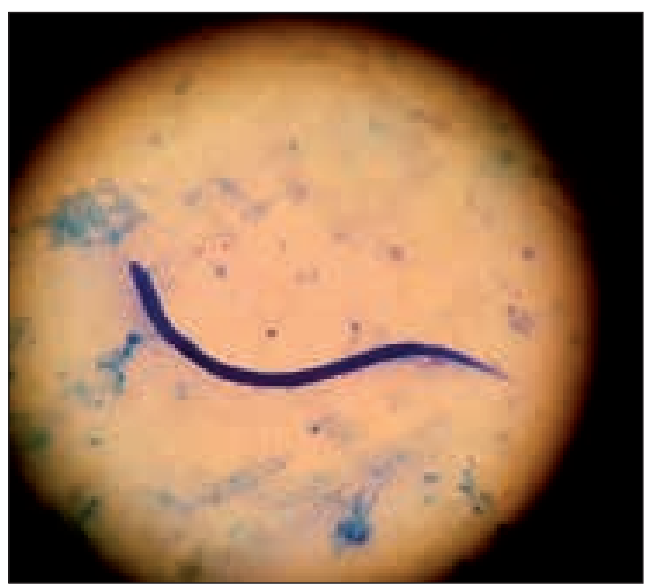

Figura 3. Caso 1: muestra de aspirado bronquial con larvas de S. stercoralis. Tinción Ziehl Neelsen 40X. diarrea, constipación y mala absorción ${ }^{[4]}$. La posibilidad de estrongiloidiasis intestinal invasiva debe ser considerada como diagnóstico diferencial de los cuadros de abdomen agudo en pacientes sometidos a terapia inmunosupresora ${ }^{[5]}$. El examen directo de las muestras de heces es un método diagnóstico poco sensible para este agente ${ }^{[6]}$. La tinción de Gram es un procedimiento útil para el diagnóstico de estrongiloidiasis pulmonar ya que puede demostrar la presencia de larvas filariformes de S. stercoralis en muestras de esputo ${ }^{[7]}$.

La incidencia de estrongiloidiosis está aumentando en muchos países en desarrollo y se facilita por los viajes internacionales y la migración y un número creciente de pacientes inmunosuprimidos ${ }^{\left[{ }^{8]}\right.}$. La hiperinfección resulta de la enorme multiplicación de larvas infectantes ysu migración en pacientes inmunodeprimidos, pero algunos autores describen el cuadro en pacientes inmunocompetentes ${ }^{[9,10]}$.

En la hiperinfección diseminada el tratamiento de estas formas graves sigue siendo un tema de controversia en pacientes con

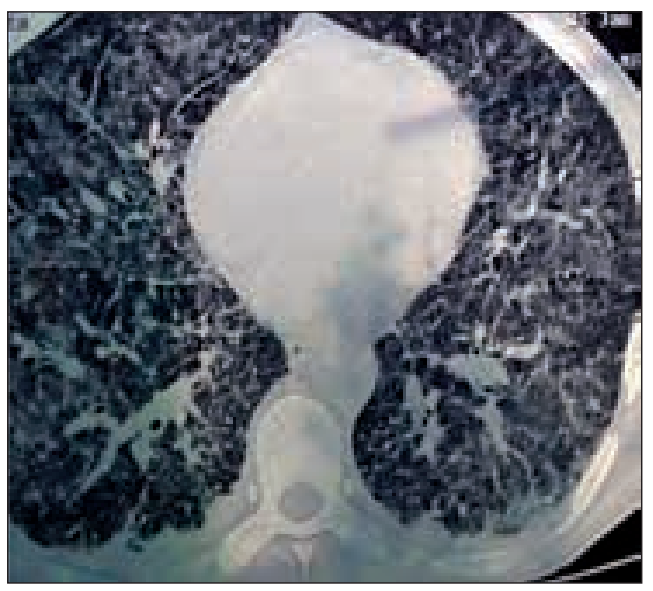

Figura 2. Caso 1: en la tomografía axial computarizada se observa el proceso inflamatorio intersticial

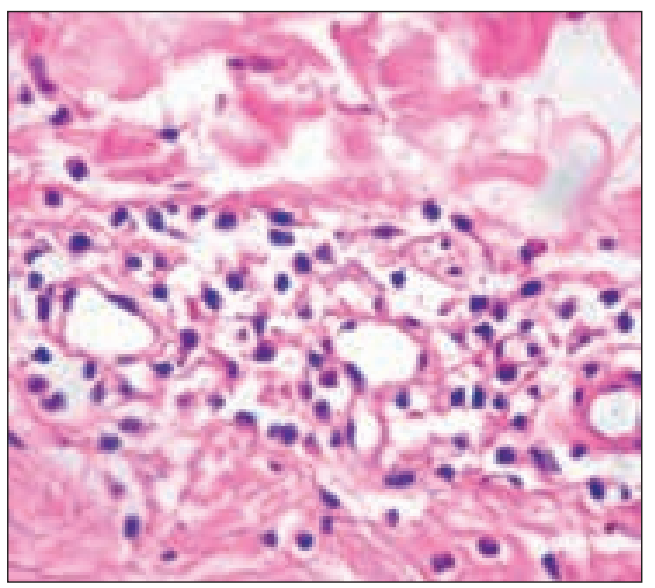

Figura 4. Caso 1: biopsia de piel en donde se observa un vasculitis leucocitoclástica. Tinción hematoxilina eosina 40X. 
reducción de la absorción intestinal de las formulaciones orales de fármacos antiparasitarios. En este caso la ruta parenteral sería la más aconsejable ${ }^{[1,12]}$. Se propone como tratamiento a la ivermectina $200 \mathrm{ug} / \mathrm{kg}$ por vía oral por-uno o dos días y alternativamente al albendazol $400 \mathrm{mg}$ dos veces al día durante siete días. Si las administraciones orales y/o rectales no son posibles, la formulación subcutánea veterinaria de la ivermectina es aconsejada ${ }^{[11]}$.

Reportamos dos casos de infección diseminada por Strongyloides stercoralis en pacientes con trasplante renal de único donante que fueron tratados con ivermectina por dos vías de administración diferentes.

\section{REPORTES DE CASOS}

\section{DONANTE}

Donante de sexo masculino de 24 años, con diagnóstico de muerte cerebral por rotura de aneurisma cerebral, antecedente inmediato de craneotomía por hipertensión intracerebral. Donante procedente de Lima, índice de masa corporal 22, creatinina 2,7 mg\%, y urea 65,2 mg\% en los análisis de procura. Administración de productos sanguíneos en mantenimiento, así como vasopresores, no presentó parada cardiaca, permaneció afebril administrándose meropenem. Familia niega antecedentes epidemiológicos de importancia.

\section{CASO 1}

Hombre de 50 años, con antecedente de enfermedad renal crónica terminal, secundaria a hipertensión arterial, en hemodiálisis por cuatro años y que fue sometido a trasplante renal en noviembre de 2014. El paciente recibió como inducción dos dosis de basiliximab $20 \mathrm{mg}$, y como tratamiento de mantenimiento tacrolimus, micofenolato y prednisona, con evolución favorable. Luego de dos meses acude al servicio de emergencia por presentar cefalea, vómitos biliosos, dolor epigástrico tipo ardor de aparición postprandial que se acentuaba con la palpación asociado a dispepsia, y estreñimiento. Luego se agrega fiebre, disnea progresiva y rash petequial difuso en región abdominal (Figura 1).

Ingresa a unidad de cuidados intensivos (UCI) por presentar insuficiencia respiratoria, evidenciándose en la tomografía pulmonar un incremento de la densidad intersticial (Figura 2).

Al examen del lavado bronquioalveolar con tinción Ziehl Neelsen se observan larvas de Strongyloides stercolaris (Figura 3), además de baciloscopia negativa. En el examen microscópico de muestra de aspirado duodenal también se observó larvas del mismo parásito. El examen microscópico para Pneumocystis jirovecii fue negativo. Durante su evolución presenta residuo gástrico alto, abdomen distendido e incremento del dolor que simula abdomen quirúrgico, y la tomografía abdominal muestra niveles hidroaéreos en asas intestinales con un diagnóstico de obstrucción intestinal, que mejora con tratamiento médico.
Los exámenes de laboratorio mostraron leucocitos $9,9 \mathrm{mil} / \mathrm{ml}$ (abastonados 13\%, segmentados 48\%, eosinófilos 3\%, linfocitos $8 \%)$, transaminasa glutámico pirúvica 45 UI, transaminasa glutámico oxalacética $18 \mathrm{UI}$, bilirrubina total $0,7 \mathrm{mg} \%$, albumina 2,4 gr\%, fosfatasa alcalina $93 \mathrm{U} / \mathrm{L}$, plaquetas $410000 \mathrm{~mm}^{3}$, hemoglobina $10,8 \mathrm{~g} \%$. La oximetría muestra un persistente gradiente oxigenatorio.

El examen anatomopatológico de piel de zona de rash describe una vasculitis leucocitoclástica y dermatitis perivascular superficial, ortoqueratosis laminar, edema leve en dermis superficial y media, e infiltrado linfomononuclear con presencia de leucocitos y algunos eosinófilos (Figura 4).

Recibió tratamiento por vía oral con ivermectina 9 mg/día más albendazol $400 \mathrm{mg}$ dos veces al día por tres días.

Seguimiento de laboratorio: fosfatasa alcalina $190 \mathrm{U} / \mathrm{L}$, transaminasa glutámico pirúvica $86 \mathrm{U} / \mathrm{L}$, transaminasa glutámico oxalacética $80 \mathrm{U} / \mathrm{L}$, gamma glutamil transpeptidasa $171 \mathrm{U} / \mathrm{L}$, bilirrubina 1,7 mg dl, bilirrubina directa 1,4 mg dl, albúmina 1,8 gr\%, BK (-), urocultivo: Candida spp >100 mil UFC/ml.

Evoluciona desfavorablemente a shock séptico refractario, falleciendo al mes de su hospitalización por neumonía asociada a ventilador en paciente inmunosuprimido e hiperinfección por Strongyloides. stercoralis.

\section{CASO 2}

Hombre de 52 años, con antecedente de enfermedad renal crónica terminal, secundaria a hipertensión arterial y diabetes mellitus 2, en hemodiálisis por cinco años quien recibió trasplante renal con donante cadavérico en noviembre de 2014 siendo receptor del mismo donante del caso 1 . Recibió como inducción dos dosis de basiliximab $20 \mathrm{mg}$, y como tratamiento de mantenimiento tacrolimus, micofenolato y prednisona; según protocolo del servicio, evolucionando favorablemente. Luego de cuatro meses postrasplante, acude a la consulta presentando astenia, disnea leve, fiebre, epigastralgia, llenura precoz y edema de miembros inferiores, motivo por el cual es hospitalizado. Al examen físico se evidencia palidez moderada, con presencia de máculas eritematovioláceas menores de $2 \mathrm{~mm}$ de diámetro en hemiabdomen superior (Figura 7), abdomen globuloso, dolor difuso leve a la palpación profunda, no visceromegalia, lesiones descamativas en región palmar de ambas manos, presión arterial $80 / 40 \mathrm{mmHg}$, frecuencia respiratoria $24 \mathrm{resp} / \mathrm{min}$, frecuencia cardiaca $100 \mathrm{lat} / \mathrm{min}$, temperatura $36,8^{\circ} \mathrm{C}$, saturación $0289 \%$; $\mathrm{FiO}_{2} 0,50 \%$, y diuresis $2000 \mathrm{~cm}^{3} /$ día. En la evaluación pulmonar: murmullo vesicular disminuido en ambas bases pulmonares, subcrépitos bibasales.

Presentó a su ingreso leucocitos de $3000 \mathrm{mil} / \mathrm{ml}$ sin eosinofilia, con hemoglobina $6 \mathrm{gr} \%$, no retención nitrogenada. Al tercer día de hospitalización cursa con insuficiencia respiratoria tipo 1 , se continúa oxígeno suplementario con máscara Venturi con valores de análisis de gases arteriales a su ingreso a 


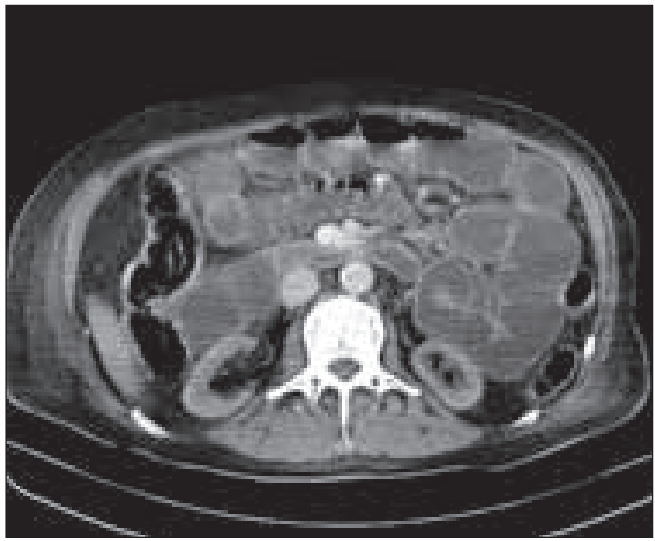

Figura 5. Caso 2: en la tomografía axial computarizada se observa la dilatación de asas intestinales.

$\mathrm{UCl}$ de $\mathrm{FiO}_{2}$ 0,5\%, $\mathrm{PaFiO}_{2}$ 121,4, G (A-a) 254,8 y deterioro oxigenatorio evolutivo que llevó a ventilación mecánica. Al décimo día presenta edema en esclavina de miembro superior derecho, la ecografía Doppler indica trombosis en vena yugular más subclavia derecha y se instaura anticoagulación con enoxaparina. Presentó melena y hematoquezia en el primer día de tratamiento llegando a tener una hemoglobina de 4,1 $\mathrm{g} \%$, reportando la endoscopía una lesión aguda de mucosa gástrica, por lo que se decide suspender la anticoagulación y sangrado cede.

Durante su evolución presenta cuadro de suboclusión Intestinal confirmado por tomografía abdominopélvica (Figura 5) que cede a manejo médico. Se recibe informe de biopsia de piel, encontrándose larvas de Strongyloides stercolaris entre las fibras de colágeno de la dermis (Figura 6). En la baciloscopia de esputo no se encontró BAAR, pero se identificó, al igual que el primer caso descrito líneas arriba, larvas de Strongyloides stercolaris.

Se inicia tratamiento por vía subcutánea con ivermectina (200 ug/kg) $12 \mathrm{mg} /$ día por siete días más albendazol $400 \mathrm{mg}$ dos veces al día por vía oral durante diez días, y meropenem $1 \mathrm{gr}$ cada ocho horas por vía endovenosa durante 14 días ${ }^{[13,14]}$. El inicio del tratamiento fue precoz, luego del diagnóstico de muestra respiratoria, suspendiendo la inmunosupresión. Presentó encefalopatía, por lo cual se realizó punción lumbar con características normales además de tomografía cerebral normal.

Al octavo día de tratamiento ya no se evidencia larvas de Strongyloides stercoralis en muestra de aspirado bronquial. Es extubado con éxito, cede fiebre luego de aproximadamente 12 días de tratamiento. Es dado de alta de UCI luego de 20 días de hospitalización. Actualmente es seguido ambulatoriamente con controles negativos para $S$. stercolaris, con función renal normal y niveles adecuados de inmunosupresión.

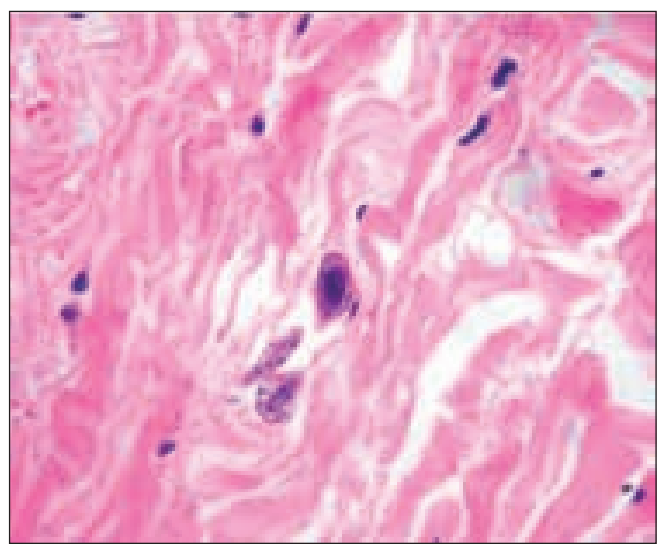

Figura 6. Caso 2: biopsia de piel con presencia de larvas de Strongyloides stercolaris entre las fibras de colágeno de la dermis. Tinción hematoxilina eosina 40X.

\section{DISCUSIÓN}

Dos casos que representan una probable trasmisión de $S$. stercolaris de un donante único y en muerte cerebral, sin aparente antecedente epidemiológico de importancia, ni eosinofilia. El historial médico, así como el trabajo previo al trasplante no tuvieron factores de riesgo de importancia en ambos receptores. El diagnóstico final de estos dos casos fue estrongiloidiasis diseminada con shock séptico asociado e insuficiencia respiratoria en pacientes inmunocomprometidos.

El inicio de síntomas gastrointestinales inespecíficos y, luego respiratorios hizo que se solicitara lavado broncopulmonar, donde se observaron larvas de S. stercolaris cuya presencia podría explicarse por el ciclo de Loos que se cumple en este tipo de parasitosis y que implica afección de diversos tejidos. A diferencia de otros casos, el hemograma no presentó eosinofilia, en ninguno de los dos casos, pero sí fue llamativa la anemia severa en el segundo caso. Durante la evolución del segundo caso en UCl este cursó con shock distributivo, deterioro ventilatorio, depresión miocárdica, oclusión intestinal, trombosis de vena yugular y subclavia derecha, además de bacteriemia por Enterococo gallinarum resistente a vancomicina ${ }^{[15]}$.

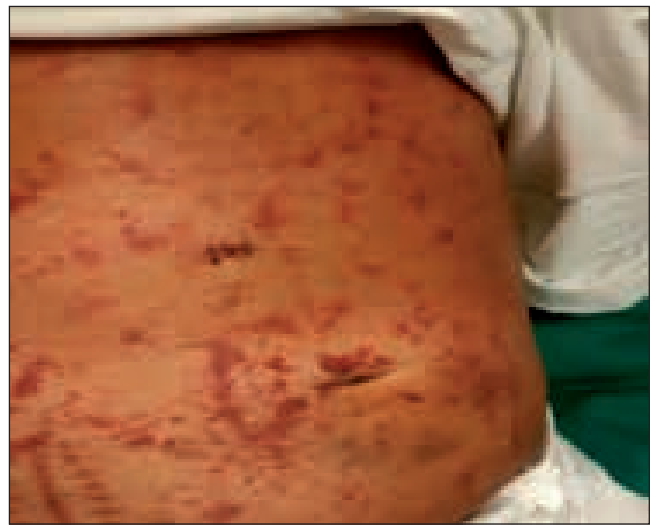

Figura 7. Caso 2: se observan lesiones purpúricas en abdomen. 
Las manifestaciones cutáneas que fueron prominentes en ambos pacientes incluyen una erupción migratoria llamada larva currens y urticaria recurrente que con mayor frecuencia afectó el abdomen. Además, en ambos pacientes hubo íleo siendo la probable causa descrita como una reacción inflamatoria de la migración de la larva de S. stercolaris al intestino delgado dentro de las glándulas mesentéricas. En esta migración la larva lleva con ella a bacterias coliformes, la más importante Escherichia coli causante de septicemia y meningitis ${ }^{[3]}$. Esta última situación clínica puede resultar en inadecuada biodisponibilidad y pobre eficacia terapéutica de preparaciones oral/enteral de tiabendazol e ivermectina.

El donante cadavérico fue la fuente probable de infección por la posibilidad del origen endémico de parasitosis conocida y muchos de los pacientes pueden no mostrar signos clínicos de infección. Sin embargo, no se pudo confirmar, debido a la no disponibilidad del expediente médico del donante. Las larvas suelen sobrevivir relativamente corto tiempo a la isquemia fría a la que los riñones son sometidos antes de ser trasplantado y podría haber sido la fuente de la infección en el receptor ${ }^{[2,16]}$.

La ivermectina es ahora reconocida como una opción terapéutica por las altas tasas de eliminación larvaria y menos efectos secundarios. Varias avermectinas veterinarias parenterales (incluida la ivermectina) se utilizan con frecuencia para el tratamiento y la profilaxis de las estrongiloidiasis gastrointestinal en ganado a una dosis de $200 \mathrm{ug} / \mathrm{kg}$; no obstante, el proceso de aprobación de medicamentos de uso veterinario es diferente a las de uso humano ${ }^{[17]}$. Las propiedades farmacocinéticas de la ivermectina pueden ser modificadas en pacientes críticamente enfermos debido a que se une en gran medida a la albumina humana; por ello, la concentración del fármaco libre y la acción terapéutica podrían estar elevadas en procesos de inflamación sistémica con hipoalbuminemia. Sin embargo, un estudio reciente encontró menos de $1 \%$ de ivermectina libre situado en el plasma, siendo una posible explicación para este hallazgo la fuerte unión entre el fármaco y alta concentración de alfa-1 glucoproteína ácida, lo que reduciría la distribución de medicamentos a los tejidos ${ }^{[12,18]}$.

S. stercoralis es endémica en todas las regiones del Perú, hiperendémica en la selva y menos endémica en las regiones andinas y costeras. El uso de la prueba de frotis directo subestima las tasas de prevalencia de S. stercoralis en Perú, por lo que se recomienda el uso rutinario de técnicas altamente sensibles cuando sea posible ${ }^{[19]}$. Asimismo, debido a que la estrongiloidiasis se ha descrito con todos los trasplantes de órganos, se debe descartar esta parasitosis antes de trasplantar órganos en áreas endémicas o en circunstancias sospechosas ${ }^{[20]}$.

Estos dos casos subrayan la necesidad en programas de trasplantes disponer de protocolo ampliado para S. stercoralis; serología usando ELISA para detectar IgG o análisis de materia fecal y debe impulsar el tratamiento precoz o profilaxis en donantes y receptores, así como la oportuna notificación de las organizaciones de obtención de órganos y centros de trasplante
[21-23]. El tamizaje de donantes vivos de órganos ofrece la oportunidad de tratar a los donantes que resultaron positivos disminuyendo el riesgo de transmisión de $S$. stercoralis ${ }^{[24]}$.

Las guías norteamericanas en trasplante renal incluyen como tamizaje el estudio del receptor $\mathrm{VIH}$, hepatitis B y C, citomegalovirus, herpes simple, varicela zoster, virus Epstein Bar, toxoplasma, sífilis y test de Mantoux ${ }^{[24]}$. Por su parte, algunas guías latinoamericanas incluyen el estudio de parásitos de acuerdo con su carga epidemiológica (T. cruzi o S. stercoralis) ${ }^{[25]}$ y las Guías de la Sociedad Americana de Trasplantes (AST) recomiendan la búsqueda de $S$. stercoralis en situación de riesgo en receptores de donantes vivos y en lo posible durante la evaluación pretrasplante de donantes fallecidos con factores de riesgo epidemiológico o eosinofilia inexplicada ${ }^{[26,2]}$.

En el caso 1 de esta revisión fue tratado tanto con ivermectina y albendazol oral en paciente con sepsis y falla multiorgánica con demostración del parásito en vía aérea y enteral persistentemente con fallo terapéutico y fallecimiento del paciente. A diferencia del caso 2 que recibió ivermectina parenteral por fallo intestinal y tuvo una evolución favorable con negativización del parásito. Lamentablemente no se disponía de pruebas de dosaje de la ivermectina en suero que permita evaluar una posible toxicidad a nivel del sistema nervioso central en el caso 2 .

En conclusión, el síndrome de diseminación e hiperinfección por S. stercoralis se debe considerar una emergencia médica potencial y el tratamiento debe iniciarse inmediatamente. Aunque no hay ensayos clínicos controlados, la droga de elección es ivermectina. La duración y ruta de administración debe ser individualizada siendo el objetivo la erradicación total del parásito y/o hasta no tener evidencia de dos semanas completas de exámenes de heces negativos. Finalmente, como parte de la vigilancia después de un trasplante renal, se recomienda la realización de exámenes continuos de esputo.

\section{REFERENCIAS BIBLIOGRÁFICAS}

1. Mendes T, Minori K, Ueta M, Miguel DC, AllegrettiSM.Strongyloidiasis current status with emphasis in diagnosis and drug research. J Parasitol Res. 2017;2017:5056314.

2. Kim JH, Kim DS, Yoon YK, Sohn JW, Kim MJ. Donor-derived strongyloidiasis infection in solid organ transplant recipients: a review and pooled analysis. Transplant Proc. 2016;48(7):2442-9.

3. Macguire J. Intestinal nematodes: Strongyloidiasis. In: Mandell GDR, Bennett J, Dolin R, editors. Mandell, Douglas, and Bennett's principles and practice of infectious diseases. New York: Elsevier/ Churchill Livingstone; 2009. p. 3582-3.

4. Bannon JP, Fater M, Solit R. Intestinal ileus secondary to Strongyloides stercoralis infection: case report and review of the literature. Am Surg. 1995;61(4):377-80.

5. Rodrigues MAM, Fróes RC, Anefalos A, Kobayasi S. Invasive enteritis by Strongyloides stercoralis presentando como distress abdominal agudo y bajo terapia con corticoids. Rev Hosp Clin. 2001;56(4):103-6. 
6. Uparanukraw F, Phongsri S, Morakote N. Fluctuations of larval excretion in Strongyloides stercoralis infection. Am J Trop Med Hyg. 1999;60(6):967-73.

7. Center for Disease Control and Prevention. Parasites - Strongyloides: resources for health professionals [Internet]. Atlanta, GA: US Department of Health and Human Resources, CDC; 2012 [citado el 15 de julio de 2017]. Disponible en: http://www.cdc.gov/parasites/ strongyloides/health_professionals/index.html

8. Wang C, Xu J, Zhou X, Li J, Yan G, James AA, Chen X. Strongyloidiasis: an emerging infectious disease in China. Am J Trop Med Hyg. 2013;88(3):420-5

9. Puthiyakunnon S, Boddu S1, Li Y1, Zhou X1, Wang C1, Li J, et al. Strongyloidiasis--an insight into its global prevalence and management. PLoS Negl Trop Dis. 2014;8(8):e3018 .

10. Shorman M, Al-Tawfiq JA. Strongyloides stercoralis hyperinfection presenting as acute respiratory failure and Gram-negative sepsis in a patient with astrocytoma. Int J Infect Dis. 2009;13(5):e288-91.

11. Marty FM, Lowry CM, Rodríguez M, Milner DA, Pieciak WS, Sinha A, et al. Treatment of human disseminated strongyloidiasis with a parenteral veterinary formulation of ivermectin. Clin Infect Dis. 2005;41(1):e5-8.

12. Rose CE, Paciullo CA, Kelly DR, Dougherty MJ, Fleckenstein LL. Fatal outcome of disseminated strongyloidiasis despite detectable plasma and cerebrospinal levels of orally administered ivermectin. J Parasitol Res. 2009(2009):818296.

13. Salluh JI, Feres GA, Velasco E, Holanda GS, Toscano L, Soares M. Successful use of parenteral ivermectin in an immunosuppressed patient with disseminated strongyloidiasis and septic shock. Intensive Care Med. 2005;31(9):1292.

14. Chiodini PL, Reid AJ, Wiselka MJ, Firming R, Foweraker J. Parenteral ivermectin in Strongyloides hyperinfection. Lancet. 2000;355(9197):43-4.

15. Sander HS. Enterococos resistentes a vancomicina: ¿̇nfección emergente inminente? Rev Chil Infectol. 2002;19/Suppl 1):S50-S55

16. Palau LA, Pankey GA. Strongyloides hyperinfection in a renal transplant recipient receiving cyclosporine: possible Strongyloides stercoralis transmission by kidney transplant. Am J Trop Med Hyg. 1997;57(4):413-5.

17. Pacanowski J, Santos MD, Roux A, LE Maignan C, Guillot J, Lavarde $V$, et al. Subcutaneous ivermectin as a safe salvage therapy in Strongyloides stercoralis hyperinfection syndrome: a case report. Am. J. Am J Trop Med Hyg. 2005;73(1):122-4.

18. Leung V, Al-Rawahi GN, Grant J, Fleckenstein L, Bowie W. Case report: failure of subcutaneous ivermectin in treating Strongyloides hyperinfection. Am J Trop Med Hyg. 2008;79(6):853-5.

19. Marcos LA, Cabrera R, Machicado JD, Canales M, Terashima A. Distribution of prevalence of Strongyloides stercoralis in Peru (1981-2010): an exploratory study. Rev Peru Parasitol. 2010;18(2):e39-e40.

20. Sadjadi SA, Damodaran C, Sharif M. Strongyloides stercoralis infection in transplanted patients. Am J Case Rep. 2013;14:205-9.

21. Abdalhanid BA, Al Abadi AM, Al Saghier MI, Joudeh AA, Shorman MA, Amr SS. Strongyloides stercoralis infection in kidney trasplant recipiejts. Saudi J Kidney Dis Transpl. 2015;26(1):98-102.

22. Le $M$, Ravin $K$, Hasan A, Clauss $H$, Muchant DG, Pasko JK, et al. Single donor-derived strongyloidiasis in three solid organ transplant recipients: case series and review of the literature. Am J Transplant. 2014;14(5):1199-206.

23. Roxby AC, Gottleib GS, Limaye AP. Strongyloidiasis in transplant patients. Clin Infect Dis. 2009;49(9):1411-23.

24. Abanyie FA, Gray EB, Delli Carpini KW, Yanofsky A, McAuliffe I, Rana $M$, et al. Donor-derived Strongyloides stercoralis infection in solid organ transplant recipients in the United States, 2009-2013. Am J Transplant. 2015;15(5):1369-75.

25. Instituto Nacional de Ciencias Médicas y Nutrición Salvador Zubirán. Protocolo de trasplante renal 2015 [Internet]. México: INCMNSZ;2015 [citado el 10 de abril de 2017]. Disponible en: http://www.innsz.mx/imagenes/ProtocoloTR-INNSZ-2015-ver-10. pdf

26. Schwartz BS, Mawhorter SD; AST Infectious Diseases Community of Practice. Parasitic infections in solid organ transplantation. Am J Transplant. 2013;13 Suppl 4:280-303.

\section{Las ediciones anteriores de Acta Médica Peruana están disponibles en:}

\section{www.scielo.org.pe}

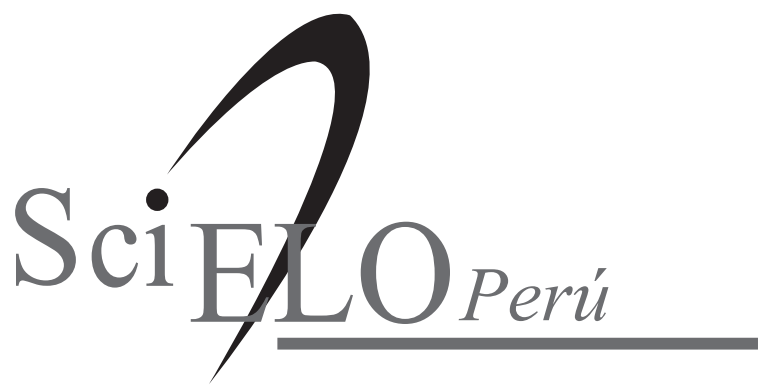

